\title{
Stereocontrolled Access to Enantiopure 7-Substituted cis- and trans-Octahydroindoles
}

\author{
Elena Ghirardi, ${ }^{\dagger}$ Rosa Griera, ${ }^{\dagger}$ Miriam Piccichè, ${ }^{\dagger}$ Elies Molins,$\stackrel{\star}{\star}$ Israel Fernández, ${ }^{\S}$ Joan Bosch,${ }^{\dagger}$ and \\ Mercedes Amat ${ }^{*}, \dagger$ \\ Laboratory of Organic Chemistry, Faculty of Pharmacy, and Institute of Biomedicine (IBUB), University of Barcelona, \\ 080028-Barcelona, Spain \\ *Institut de Ciència de Materials de Barcelona (ICMAB, CSIC), Campus UAB, 08193-Bellaterra, Spain \\ §Departamento de Química Orgánica I, Facultad de Ciencias Químicas, Centro de Innovación en Química Avanzada \\ (ORFEO-CINQA), Universidad Complutense de Madrid, 28040-Madrid, Spain
}

amat@ub.edu

Supporting Information Placeholder

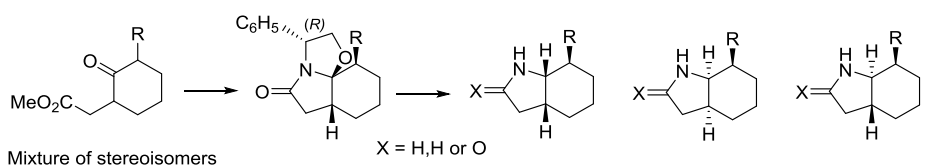

\footnotetext{
ABSTRACT: Cyclocondensation of $(R)$-phenylglycinol with stereoisomeric mixtures (racemates, cis/trans) of 3-substituted 2oxocyclohexaneacetates stereoselectively afforded tricyclic oxazoloindolone lactams, from which straightforward procedures for the stereocontrolled formation of enantiopure 7-substituted octahydroindoles with a variety of stereochemical patterns have been developed. The methodology has been successfully applied to the synthesis of (+)- $\alpha$-lycorane.
}

Phenylglycinol- and other aminoalcohol-derived oxazolopiperidone and oxazoloquinolone lactams have proven to be versatile building blocks for the enantioselective synthesis of a wide variety of diversely substituted piperidine-containing heterocycles, including complex alkaloids belonging to different skeletal types. ${ }^{1}$ The usefulness of these lactams lies in their easy preparation, by stereoselective cyclocondensation of the chiral nonracemic aminoalcohol with an appropriate $\delta$-oxo acid derivative, and in their functionalization and conformational rigidity, which allow the stereocontrolled formation of $\mathrm{C}-\mathrm{C}$ bonds at the different positions of the nitrogen heterocycle.

Starting from 2-oxocyclohexanepropionate-based $\delta$-keto esters, the cyclocondensation stereoselectively affords tricyclic oxazoloquinolone lactams, which can be easily converted to enantiopure $c i s$-decahydroquinoline alkaloids. ${ }^{2}$ Depending on the structural characteristics of the substrate, the cyclocondensation involves a dynamic kinetic resolution and/or differentiation of enantiotopic or diastereotopic ester groups.

Bearing in mind that the octahydroindole nucleus is present in numerous natural bioactive compounds, for instance Amaryllidaceae alkaloids, we considered expanding the scope of the above stereoconvergent cyclocondensation reactions towards the generation of tricyclic oxazoloindolone lactams as precursors of this nucleus. This would require starting from appropriately substituted 2-oxocyclohexaneacetate derivatives $(\gamma$ - instead of $\delta$-keto esters).

In this letter, we report a general straightforward procedure for the stereocontrolled access to enantiopure 7-substituted cis- and trans-octahydroindoles. The few precedents of the enantioselective synthesis of 7-substituted octahydroindoles all deal with 7-aryl cis-derivatives, ${ }^{3}$ used as intermediates in the synthesis of lycorane-like structures.

Scheme 1. Envisaged Access to Enantiopure 7-Substituted Octahydroindoles

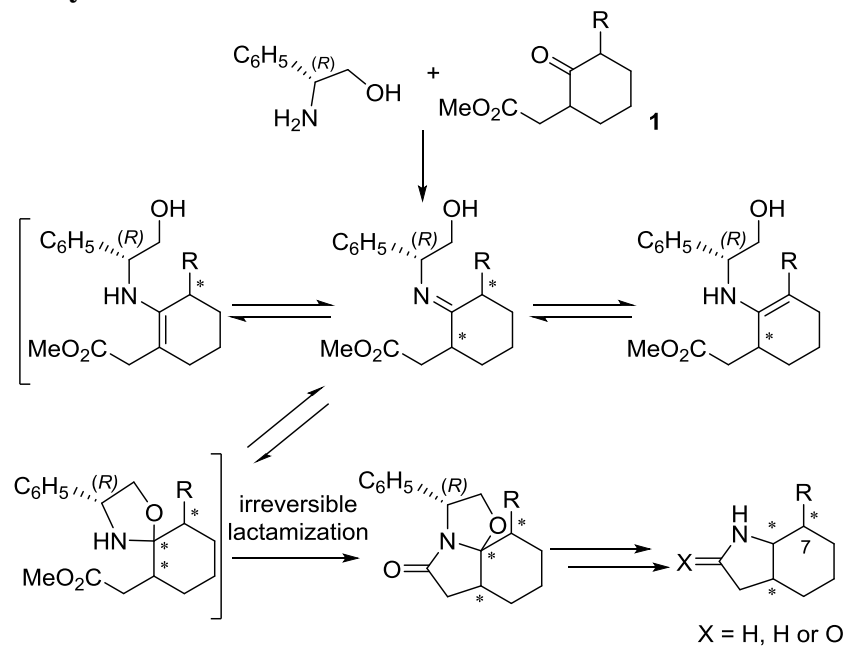

The preparation of the target enantiopure octahydroindoles was envisaged as outlined in Scheme 1. Starting from a stereoisomeric mixture of 3-substituted 2-oxocyclohexane-acetates 1 (two racemates when $\mathrm{R}=$ alkyl or aryl; one racemate and a 
meso form when $\mathrm{R}=\mathrm{CH}_{2} \mathrm{CO}_{2} \mathrm{Et}$ ), cyclocondensation with $(R)$ phenylglycinol would afford four stereoisomeric imines, which would be in equilibrium through the corresponding enamines with eight stereoisomeric oxazolidines. A final irreversible lactamization would afford the target enantiopure tricyclic lactams. The stereoselectivity of the process would depend upon the kinetically controlled lactamization step.

Accordingly, cyclocondensation of $\gamma$-keto ester 1a (mixture of two racemates) with $(R)$-phenylglycinol in the presence of AcOH in refluxing benzene afforded a single 10-substituted tricyclic lactam 2a in $75 \%$ yield. Minor amounts $(8 \%)$ of unsaturated bicyclic lactam 3a were also formed (Scheme 2). Similar stereoconvergent cyclocondensation reactions occurred starting from $\gamma$-keto esters $\mathbf{1 b}$-d (two racemates) or $\mathbf{1 e}$ (mixture of one racemate and a meso form), leading to the respective tricyclic lactams $\mathbf{2} \mathbf{b}-\mathbf{e}$, also accompanied by the corresponding unsaturated lactams $\mathbf{3}$ in series $\mathbf{b}, \mathbf{c}$, and e (but not $\mathbf{d}$, when $\left.\mathrm{R}=\mathrm{C}_{6} \mathrm{H}_{5}\right)$ as byproducts ( $8-13 \%$ yield). The absolute configuration of $\mathbf{2 a}, \mathbf{2 d}$, and $\mathbf{3 a}$ was unambiguously established by X-ray crystallographic analysis. ${ }^{4}$

\section{Scheme 2. Stereoconvergent Cyclocondensation Reactions}
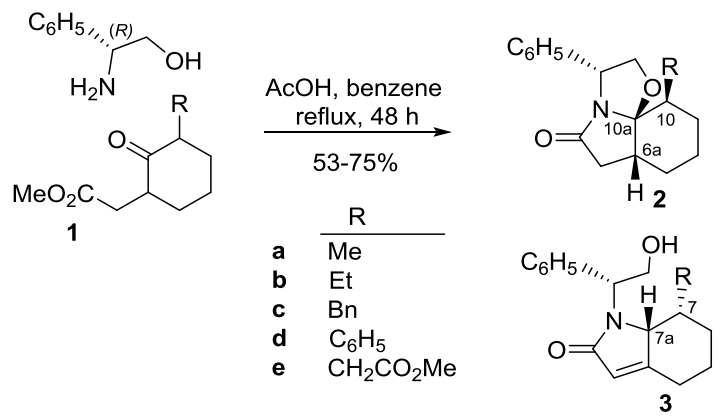

The stereoselective formation of $\mathbf{2 a - e}$ can be rationalized by considering that steric constraints prevent the formation of transfused tricyclic lactams ${ }^{5}$ and that irreversible lactamization from the equilibrating mixture of oxazolidines occurs faster from oxazolidine $\mathbf{A}$, in which the substituent $\mathrm{R}$ on the cyclohexane ring is equatorial and the carboxylate approaches the nitrogen atom from the less hindered face, opposite to the phenyl, thus defining the configuration of the $6 a$ and $10 \mathrm{a}$ stereocenters (Scheme 3). Indeed, our calculations indicate that the cyclization reactions involving intermediate $\mathbf{A}(\mathrm{R}=\mathrm{Me}$ or $\mathrm{Ph})$ are kinetically $\left(\Delta \Delta \mathrm{G}^{\neq} \approx 5\right.$ $\mathrm{kcal} / \mathrm{mol})$ and also thermodynamically $\left(\Delta \Delta \mathrm{G}_{\mathrm{R}} \approx-5.5\right.$ $\mathrm{kcal} / \mathrm{mol}$ ) favored over those processes where the R substituent is axial (see Figure S1 in the Supporting Information). ${ }^{4}$

\section{Scheme 3. Irreversible Lactamization Step}

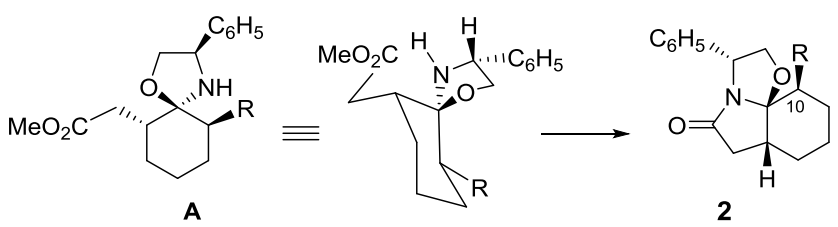

In turn, unsaturated lactams $\mathbf{3}$ would result from tricyclic lactams 10-epi-2, transiently formed during the cyclocondensation reaction. The opening of the oxazolidine ring of 10-epi-
2 under the acidic reaction conditions would be followed by isomerization of the double bond in the resulting $N$-acyl iminium species, with a final protonation from the less hindered face to give the 7-H/7a-H cis isomers $\mathbf{3}$, as outlined in Scheme 4.

\section{Scheme 4. Formation of Unsaturated Lactams 3}

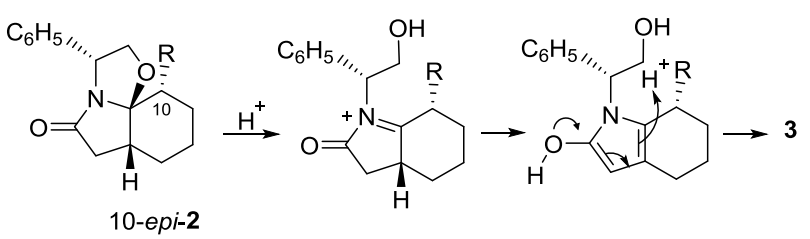

This hypothesis was confirmed when a mixture of lactams 2a and 10-epi-2a, prepared by catalytic hydrogenation of unsaturated tricyclic lactam $\mathbf{5}$, was converted into a mixture of recovered lactam 2a and unsaturated lactam 3a after heating $\left(\mathrm{C}_{6} \mathrm{H}_{6}\right.$, reflux, AcOH) (Scheme 5). The required lactam 5 was prepared by cyclocondensation of $(R)$-phenylglycinol with cyclohexenone-based $\gamma$-keto ester 4 . The lower stability of tricyclic lactams 10-epi-2 compared with their C-10 epimers 2 can be explained by the occurrence of destabilizing 1,3-axial interactions caused by the axial C-10 substituent in 10-epi-2.

The fact that phenyl-substituted bicyclic lactam 3d was not observed as a byproduct in the cyclocondensation reaction indicates that tricyclic lactam 10-epi-2d was not formed in the process. Again, this is supported by theoretical calculations, which suggest that equilibration of oxazolidine A (Scheme 3) to the corresponding epimer bearing an axial $\mathrm{R}$ substituent is energetically disfavored when $\mathrm{R}$ is phenyl (see Supporting Information).

Scheme 5. Oxazolidine Ring Opening from Tricyclic Lactam 10-epi-2a

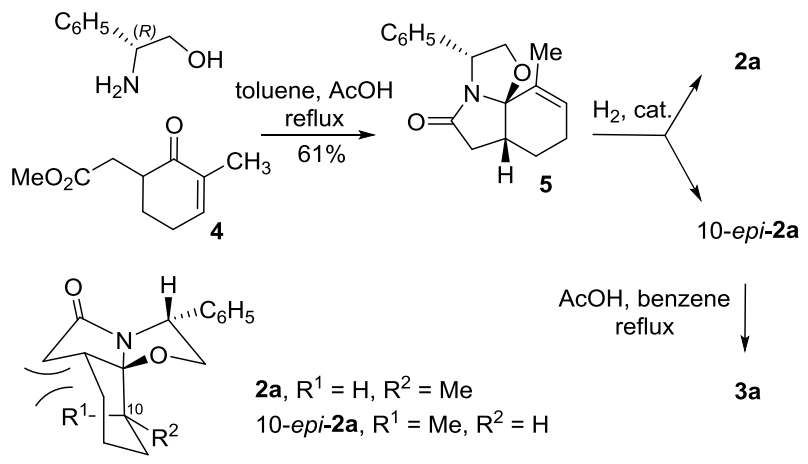

We then explored the synthetic potential of lactams 2 in the synthesis of enantiopure octahydroindole derivatives. Alane reduction of 2a-e brought about both the reduction of the lactam carbonyl and the reductive cleavage of the oxazolidine ring, with retention of configuration (see $\mathbf{B}$ in Scheme 6), to give cis-fused octahydroindoles 6a-e and, after catalytic debenzylation, 7a-e (3a-H/7a-H/7-H cis,trans series). The same stereochemical pattern resulted from the chemoselective reduction of 2a-e with $\mathrm{Et}_{3} \mathrm{SiH}$ (or $\mathrm{Ph}_{3} \mathrm{SiH}$ )/ $\mathrm{TiCl}_{4}$ (see $\mathbf{C}$ in Scheme 6), affording cis-octahydroindolones 8a-e ${ }^{6}$ and, after $\mathrm{Na} /$ liq. $\mathrm{NH}_{3}$-promoted debenzylation, 9a-e ${ }^{7}$ (Scheme 6). 


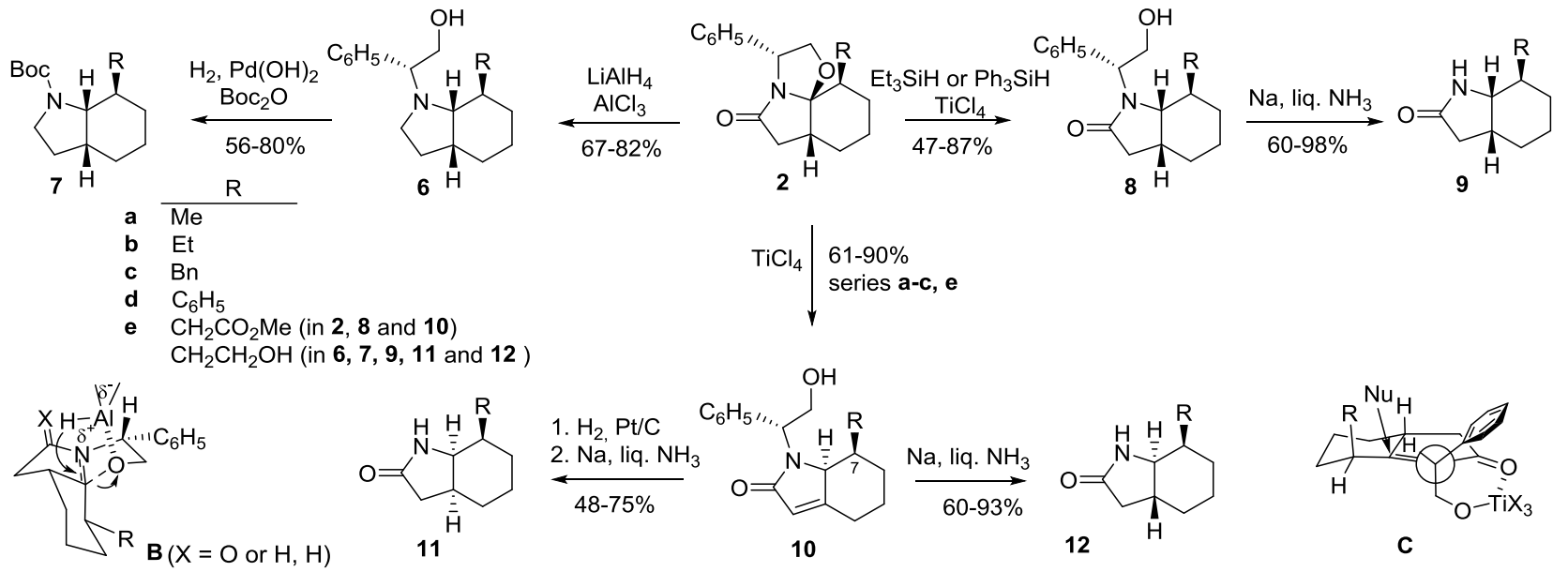

Alternatively, treatment ( $\mathrm{rt}, 18 \mathrm{~h}$ ) of tricyclic lactams $\mathbf{2 a - e}$ with $\mathrm{TiCl}_{4}$ provided either unsaturated bicyclic lactams $\mathbf{1 0}$ (series a-c,e) or, somewhat surprisingly, the 7a-epimer $\mathbf{1 3}$ in the phenyl series (Scheme 7). Catalytic hydrogenation of 10ac,e followed by $\mathrm{Na} /$ liq. $\mathrm{NH}_{3}$-promoted debenzylation gave cisoctahydroindolones 11 (3a-H/7a-H/7-H cis, cis series), whereas direct treatment of $\mathbf{1 0}$ with $\mathrm{Na} /$ liq. $\mathrm{NH}_{3}$ caused debenzylation and simultaneous reduction of the $\mathrm{C}-\mathrm{C}$ double bond, providing trans-octahydroindolones 12 (trans, cis series). Similar transformations from the phenyl-substituted unsaturated lactam 13 led to cis- and trans-octahydroindolones 9d (cis,trans series) and 14 (trans, trans series), respectively (Scheme 7). ${ }^{7}$

Scheme 7. Access to Enantiopure cis- and trans-7Aryloctahydroindoles



The relative stereochemistry of $\mathbf{1 1}$ and $\mathbf{1 2}$ was confirmed by the conversion of the minor unsaturated lactams $\mathbf{3 a - c}, \mathbf{3 e}$ to ent-11 and ent-12, as outlined in Scheme 8.

An explanation for the different stereochemical outcome of the $\mathrm{TiCl}_{4}$-promoted opening of the oxazolidine ring observed in the phenyl series is that, in all cases (series a-e), the initially formed $N$-acyl iminium species are in equilibrium with the corresponding enamides and 2-hydroxypyrroles (dienols), which undergo a kinetic protonation on the less hindered face to give the 7-H/7a-H cis-unsaturated lactams 10. A subsequent in situ equilibration takes place in the phenyl series, leading to the most stable trans isomer $\mathbf{1 3}$ (equatorial C-7 phenyl substituent) ${ }^{8}$ which is stabilized by intramolecular $\pi-\pi$ interactions (see Figure S2 in the Supporting Information).

\section{Scheme 8. Confirmation of the Stereochemistry}

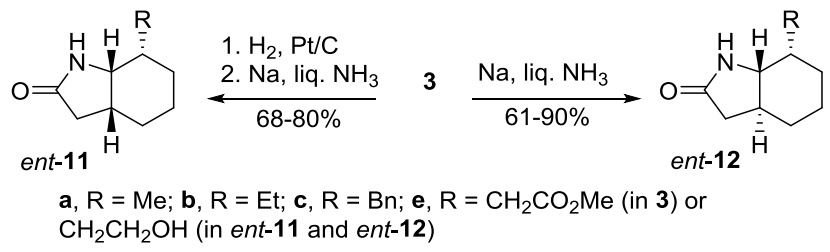

To further illustrate the usefulness of the methodology herein developed, we applied it to the synthesis of $(+)$ - $\alpha$-lycorane, which can be envisaged as a 7-aryl substituted cisoctahydroindole bearing an additional methylene bridge that connects the nitrogen atom with the aromatic ring.

The required starting $\gamma$-keto ester $\mathbf{1 5}$ (two racemic diastereoisomers), ${ }^{9}$ which incorporates a (methylenedioxy)phenyl substituent, reacted with $(R)$-phenylglycinol under the usual cyclocondensation reaction conditions to stereoselectively afford a single tricyclic lactam, 16, with generation of three stereogenic centers of a well-defined configuration. A subsequent alane reduction followed by debenzylation stereoselectively afforded cis-7-aryloctahydro-indole 17, which was converted to the target $(+)$ - $\alpha$-lycorane by a final reaction with formaldehyde (Scheme 9). ${ }^{10}$ 




In conclusion, cyclocondensation of $(R)$-phenylglycinol with stereoisomeric mixtures (racemates, cis/trans) of 3-substituted 2-oxocyclohexaneacetates stereoselectively provides tricyclic oxazoloindolone lactams in a stereoconvergent process involving a dynamic kinetic resolution of the racemic substrates. Further stereocontrolled transformations open straightforward routes to enantiopure 7-substituted octahydroindole derivatives bearing a variety of stereochemical patterns.

\section{REFERENCES}

(1) For reviews, see: (a) Escolano, C.; Amat, M.; Bosch, J. Chem. Eur. J. 2006, 12, 8198. (b) Amat, M.; Pérez, M.; Bosch, J. Synlett 2011, 143. (c) Amat, M.; Pérez, M.; Bosch, J. Chem. Eur. J. 2011, 17, 7724. For reviews covering pioneering work in the field, see: (d) Romo, D.; Meyers, A. I. Tetrahedron 1991, 47, 9503. (e) Meyers, A. I.; Brengel, G. P. Chem. Commun. 1997, 1. (f) Groaning, M. D.; Meyers, A. I. Tetrahedron 2000, 56, 9843.

(2) (a) Amat, M.; Griera, R.; Fabregat, R.; Molins, E. Bosch, J. Angew. Chem. Int. Ed. 2008, 47, 3348. (b) Amat, M.; Fabregat, R.; Griera, R.; Bosch, J. J. Org. Chem. 2009, 74, 1794. (c) Amat, M.; Fabregat, R.; Griera, R.; Florindo, P.; Molins, E.; Bosch, J. J. Org. Chem. 2010, 75, 3797. (d) Amat, M.; Navío, L.; Llor, N.; Molins, E.; Bosch, J. Org. Lett. 2012, 14, 210. (e) Amat, M.; Ghirardi, E.; Navío, L.; Griera, R.; Llor, N.; Molins, E.; Bosch, J. Chem. Eur. J. 2013, 19, 16044. (f) Amat, M.; Pinto, A.; Griera, R.; Bosch, J. Chem. Commun. 2013, 49, 11032.

(3) (a) Rh-catalyzed asymmetric nitroallylation of arylmetallics: Dong, L.; Xu, Y.-J.; Yuan, W.-C.; Cui, X.; Cun, L.-F.; Gong, L.-Z. Eur. J. Org. Chem. 2006, 4093. (b) Organocatalytic enantioselective construction of nitrocyclohexanones: Rajkumar, S. Shankland, K.; Brown, G. D.; Cobb, A. J. A. Chem. Sci. 2012, 3, 584. (c) Asymmetric bifunctional thiourea-catalyzed cascade reaction: Wang, Y.; Luo, Y.-C; Zhang, H.-B.; Xu, P.-F. Org. Biomol. Chem. 2012, 10, 8211. (d) Asymmetric catalytic enantioselective conjugate addition: Sun, Z.; Zhou, M.; Li, X.; Meng, X.; Peng, F.; Zhang, H.; Shao, Z. Chem. Eur. J. 2014, 20, 6112. (e) Meng, X.-L.; Liu, T.; Sun, Z.-W.; Wang, J.-C.; Peng, F.-Z.; Shao, Z.-H. Org. Lett. 2014, 16, 3044.

(4) CCDC 1504443, 1504444, and 1504442 contain the supplementary crystallographic data for compounds $\mathbf{2 a}, \mathbf{2 d}$, and $\mathbf{3 a}$, respectively. This data can be obtained free of charge from the Cambridge Crystallographic Data Centre via www.ccdc.cam.ac.uk/data_request/cif.
(5) For related aminoalcohol-derived cis-oxazoloindolone lactams lacking additional stereocenters at the indolone moiety, see: (a) Ragan, J. A.; Claffey, M. C. Heterocycles 1995, 41, 57. (b) Ennis, M. D.; Hoffman, R. L.; Ghazal, N. B.; Old, D. W.; Mooney, P. A. J. Org. Chem. 1996, 61, 5813. (c) Allin, S. M.; James, S. L.; Elsegood, M. R. J.; Martin, W. P. J. Org. Chem. 2002, 67, 9464. (d) Jida, M.; DeprezPoulain, R.; Malaquin, S.; Roussel, P.; Agbossou-Niedercorn, F.; Deprez, B.; Laconde, G. Green Chem. 2010, 12, 961.

(6) Minor amounts of the respective C-7 epimers were observed in some runs.

(7) The relative configurations of $\mathbf{9 d}$ and $\mathbf{1 4}$ were confirmed by their ${ }^{1} \mathrm{H}$ and ${ }^{13} \mathrm{C}$ NMR data, which coincided with those reported for rac-9d and rac-14: Yasuhara, T.; Nishimura, K.; Osafune, E.; Muraoka, O.; Tomioka, K. Chem. Pharm. Bull. 2004, 52, 1109.

(8) The opposite facial protonation in the conversion of 7-methyl- or7-aryl-4,5,6,7-tetrahydroindol-2(3H)-ones to the corresponding $\alpha, \beta$ unsaturated lactams, leading to either 7-H/7a-H cis (from 7-methyl) or trans (from 7-aryl) isomers, has previously been reported: (a) Umezawa, B.; Hoshino, O.; Sawaki, S.; Sato, S.; Numao, N. J. Org. Chem. 1977, 42, 4272. (b) Endo, Y.; Shudo, K. Heterocycles 1992, $33,91$.

(9) Keto ester 15 (3:1 cis:trans ratio) was prepared in four steps from 2-cyclohexenone: Liu. C.; Xie, J.-H.; Li, Y.-L.; Chen, J.-Q.; Zhou, Q.L. Angew. Chem. Int. Ed. 2013, 52, 593.

(10) For the conversion of the Amaryllidaceae alkaloid (-)-lycorine to (-)- $\alpha$-lycorane, see: (a) Fales, H. M.; Wildman, W. C. J. Am. Chem. Soc. 1958, 80, 4395. (b) Takeda, K.; Kotera, K.; Mizukami, S.; Kobayashi, M. Chem. Pharm. Bull. 1960, 8, 483. For previous enantioselective synthesis of $\alpha$-lycorane see: c) Li, G.; Xie, J.-H.; Hou, J.; Zhu, S.-F.; Zhou, Q.-L. Adv. Synth. Catal. 2013, 355, 1597. See also refs $3 \mathrm{c}, \mathrm{d}, \mathrm{e}$.

Acknowledgment. Financial support from the MINECO/FEDER (Projects CTQ2013-44303-P, CTQ2014-51912-REDC, and CTQ2015-65384-R) and the Generalitat de Catalunya (Grant 2014SGR-155) is gratefully acknowledged. We also acknowledge the networking contribution from the COST Action CM1407. Thanks also due to the MECD (Spain) for a fellowship to E. G., the Serra Hunter programme (R. G.), and the Unipharma Graduates Project for a mobility grant to M. P.

Supporting Information Available: Complete experimental procedures and copies of ${ }^{1} \mathrm{H}$ and ${ }^{13} \mathrm{C}$ spectra of all new compounds. Crystallographic data for compounds $\mathbf{2 a}, \mathbf{2 d}$ and 3a. Figures S1 and S2, computational details, Cartesian coordinates $(\AA)$, and free energies of all the stationary points discussed in the text. This material is available free of charge via internet at http://pubs.acs.org. 